Pbilosophia Philosophia Scientiæ

Scientie Travaux d'histoire et de philosophie des sciences

10-1 | 2006

Jerzy Kalinowski : logique et normativité

\title{
Premières réflexions sur les rapports entre la logique symbolique et l'informatique
}

\section{R. Bozzi}

Traducteur : Michel Bastit et Agnès Bastit

\section{OpenEdition \\ Journals}

Édition électronique

URL : http://journals.openedition.org/philosophiascientiae/496

DOI : $10.4000 /$ philosophiascientiae.496

ISSN : $1775-4283$

Éditeur

Éditions Kimé

Édition imprimée

Date de publication : 1 avril 2006

Pagination : 117-135

ISBN : 2-84174-392-6

ISSN : $1281-2463$

Référence électronique

R. Bozzi, «Premières réflexions sur les rapports entre la logique symbolique et l'informatique », Philosophia Scientiæ [En ligne], 10-1 | 2006, mis en ligne le 10 juin 2011, consulté le 18 janvier 2021. URL : http://journals.openedition.org/philosophiascientiae/496 ; DOI : https://doi.org/10.4000/ philosophiascientiae.496 


\title{
Premières réflexions sur les rapports entre la logique symbolique et l'informatique
}

\author{
R. Bozzi \\ Université Grégorienne/Rome \\ Michel et Agnès Bastit (traducteurs) \\ Universités de Dijon et Metz
}

Résumé : Georges Kalinowski, dans « Raison, entendement et philosophie » [Kalinowski 1974, 125-127], isolait quatre fonctions d'une même faculté : l'intellect comme fonction de la connaissance intuitive, la raison (des anciens) comme fonction de la connaissance médiate, l'entendement fonction d'élaboration des sciences (au sens moderne) et la raison (des modernes) fonction d'élaboration de la philosophie ou au moins d'une partie de la philosophie. Kalinowski reconnaissait ainsi à la pensée ancienne et à la pensée moderne des contributions originales différentes. Le présent essai veut être un hommage à Georges Kalinowski, dans l'esprit de ces précisions : il donne lieu aujourd'hui, avec une brève introduction sur les rapports entre la logique de Boole et de Frege et les fondements de l'informatique, à une méditation que l'on prévoit beaucoup plus vaste sur les rapports entre logique aristotélico-thomiste, logique symbolique, mathématique, théorie des ensembles et informatique. 
I. Si nous regardons globalement la signification de la science moderne nous devons constater que le problème surgit quand on prend conscience que l'ancien concept de science, comme connaissance par le moyen de causes universelles et nécessaires est trop restrictif. N'y a-t-il pas de lois qui puissent être, certes vastes, mais non absolument universelles? Et ceci tant dans le monde physique (les lois que Copernic, Kepler, Galilée, Newton discutent) que dans le monde social (on pense à la conception de l'état de Machiavel), comme aussi dans les phénomènes évolutifs (les ères géologiques, le futur transformisme darwinien, l'histoire humaine). Voilà précisément le problème : est-ce qu'est science ce complexe de trois types de lois applicables à une série de cas empiriques : 1) les lois physiques, vastes mais non absolues; 2) les lois socio-psychologiques; 3) les lois vectorielles (par exemple celles de Maritain)? Tout ceci ensemble est histoire et environnement du devenir physique et social, tant dans son aspect général que dans l'évolution individuelle. De quoi naît le problème? L'ancienne tentative de saisir l'essence ne semble plus compatible avec l'idéal des temps nouveaux (la domination du réel). Il suit de ceci que le nouveau style de travail est purement factuel, synthétique. Machiavel ne veut pas savoir ce qu'est l'Etat, mais comment l'Etat naît, croît, périt; Léonard invoque l'expérience, Galilée propose la traduction du comportement en termes mathématiques ... Les lois, comme on l'a dit, ne sont plus essentialistes et donc universelles (c'est-à-dire applicables de fait et de droit à leurs sujets), mais factuelles-synthétiques, c'est-à-dire applicables de fait et non de droit à une série constante et homogène d'évènements (lois générales), ou applicables à des évènements hétérogènes, toujours par voie de fait (on peut avoir de cette manière une loi synthétique individuelle). Il s'ensuit que de telles lois, saisissant des rapports homogènes ou hétérogènes purement factuels, saisissent seulement des aspects partiels (une meilleure connaissance des évènements apporte intégrations et corrections) et provisoires (précisément parce que partiels), subjectifs (non pas dans le sens d'arbitraires mais dans le sens que l'on choisit facilement les aspects du réel adaptés : on pense, par exemple, aux buts bellicistes comme impulsion des conquêtes scientifiques), toujours probables (de telles lois ne donnent jamais une certitude absolue). C'est ce que résume Daniel Bell : «Un schéma conceptuel choisit des attributs particuliers d'une réalité complexe et les groupe sous une rubrique commune afin de distinguer des similitudes et des différences. En sa qualité de dispositif de mise en ordre logique, un schéma conceptuel n'est ni vrai ni faux mais seulement utile ou non ${ }^{1}$.

\footnotetext{
${ }^{1}$ Bell 1976, 9]. Les idées exprimées dans ce texte se trouvent plus amplement développées chez [Bozzi 1996, 37 ss.].
} 
N'importe quelle étude sur les méthodes des sciences indique les contributions des divers penseurs, de Léonard à Machiavel, Bacon, Galilée, Descartes, Locke ... Il est opportun de souligner ici seulement quelques-uns des aspects intéressants pour notre propos, et précisément

- le refus de la connaissance essentialiste;

- la présence de la même méthodologie dans les divers champs de recherche, moyennant les différences obligées;

- un entassement d'éléments particuliers ou universels en tant qu'éléments simples; par exemple « soleil », «terre», « arbre», «rond », 《carré », 《pensée », « être » sont posés sur le même plan par la logique de Port-Royal ${ }^{2}$, de même aussi que la «blancheur», la «dureté », la «douceur», la «pensée », le «mouvement», «homme», 《éléphant », « armée », « ébriété »... par Locke ${ }^{3}$;

- pour divers auteurs (Galilée, Descartes, Leibniz ...), la mathématique devient le modèle cognitif par lequel on peut dépasser la subjectivité de nos jugements sur la distance entre les lieux, la durée des évènements, le poids des corps, l'évaluation des évènements ${ }^{4} \ldots$

- et ceci parce que la mathématique est une recherche d'ordre et de mesure ${ }^{5}$ ou science de la quantité ${ }^{6}$. La mathématique comme science des individualités homogènes, ou des éléments considérés comme tels, domine l'interprétation du réel (par exemple : on peut

\footnotetext{
${ }^{2}$ [Arnauld \& Nicole 1969, 104].

${ }^{3}$ [Locke 1951, 123-125].

${ }^{4}$ Prenons un sac. Est-il lourd? Oui, dira un enfant; non, répond l'adulte. Mais comment un sac identique peut-il être en même temps lourd et léger? Platon aura de la peine à expliquer comment il est possible qu'un sac puisse "participer" à la fois au lourd et au léger. Aristote posera la règle sociale de l'appréciation collective à laquelle se tient encore le sens commun d'aujourd'hui : ce sac est léger, il « semblera » lourd à l'enfant et au malade, mais en soi il ne l'est pas... « En dénonçant cette illusion, la physique mathématique se construisait un monde de données quantitatives vraiment réelles, ne relevant ni de l'intelligible platonicien (sensible transféré) ni de celui d'Aristote (social cristallisé). Eh bien, à ces données quantitatives appliquons l'instrument mathématique, la mesure. Nous dirons alors que ce sac pèse, par exemple, dix kilos ...» [Lenoble 1969, 381-382].

${ }^{5}$ « Toutes les sciences qui ont pour but la recherche de l'ordre et de la mesure se rapportent aux mathématiques. »

6"Mathesis universalis est scientia de quantitate in universum, seu de ratione aestimandi... hinc fit ut mathesis universalis sit scientia de mensurae repetitione seu de numero" (Leibniz, Math.Schriften, Gerhardt, III, 53). En résumé, [Abbagnano 1964, 543-546] distingue quatre définitions fondamentales de la mathématique : pour la première, en gros jusqu'à Kant, la mathématique est science de la quantité. Pour [Odifreddi 2000, 26] "en substance, la mathématique classique se réduisait à quatre domaines, respectivement voués à l'étude du discret et du continu, ou des nombres et des figures : arithmétique et algèbre d'un côté, géométrie et analyse de l'autre. »
} 
dire une, deux, trois chaises, mais il est impossible de dire que font deux une table et une chaise, à moins que l'on ne considère qu'elles possèdent une quelconque homogénéité, en les évaluant donc comme deux réalités).

II. A) Boole écrit : « Le but de ce traité est de rechercher les lois fondamentales de ces opérations de l'esprit au moyen desquelles s'effectue le raisonnement; de leur donner une expression dans le langage symbolique d'un calcul et d'instituer, sur ce fondement, la science de la logique en en constituant la méthode ... $\gg^{7}$. Et peu après : «Néanmoins, s'il est vrai que les arguments développés dans certaines parties de cette œuvre ont déjà été traités par d'autres auteurs, j'estime que sa conception générale, la méthode qui y est employée et, dans une mesure non négligeable, les résultats auxquels celle-ci permet de parvenir, sont originaux $»^{8}$. Et quand il doit réaliser son projet Boole «propose ... de donner des expressions aux lois fondamentales du raisonnement dans le langage symbolique d'un calcul. A ce propos, il suffira de dire que les lois exprimées en langage ordinaire sont telles qu'elles suggèrent ce mode d'expression et qu'elles le rendent particulièrement et exclusivement adapté aux buts que nous nous sommes proposés ici. Non seulement il existe une étroite analogie entre les opérations que l'esprit effectue quand il fait des raisonnements généraux et ceux qu'il effectue dans la science particulière de l'algèbre : il y a encore, dans une mesure considérable, une exacte concordance entre les lois en vertu desquelles s'effectuent les deux classes d'opérations $\gg^{9}$. Formes et processus logiques fondamentaux sont mathématiques : «Il n'est pas essentiel à la mathématique de traiter avec les idées de nombre et de quantité $\gg^{10}$.

Concrètement, «toutes les opérations du langage, en tant qu'instrument du raisonnement, peuvent être conduites par le moyen d'un système de signes composés des éléments suivants :

1) Des lettres symboliques, comme $x, y$, etc., qui représentent les choses en tant qu'objets de nos actes de conception.

2) Des signes d'opérations comme,,$+- \times$, qui remplacent ces opérations de l'esprit par le moyen desquelles les conceptions des choses se trouvent combinées ou décomposées de façon à former de nouvelles conceptions qui contiennent les mêmes éléments.

3) Le signe d'identité : =

\footnotetext{
${ }^{7}$ [Boole 1976, 9].

8 [Boole 1976, 11].

9 [Boole 1976, 15].

${ }^{10}$ [Boole 1976, 24].
} 
«Et dans leur usage ces symboles sont soumis à des lois définies qui concordent en partie avec les lois des symboles algébriques et en partie en diffèrent $\gg^{11}$. On introduit de cette manière en logique une symbolique mathématique pour la conjonction $(\times)$ et l'alternative $(+)$ : c'est le début de la formulation des 16 connecteurs successivement élaborés. C'est ainsi encore que Frege, à la fin du dix-neuvième siècle, étend la fonction, centrale en mathématique, à la logique. Néanmoins, avec ces impositions, nous sommes bien loin de la mathématique, contrairement aux intentions et aux désirs clairement exprimés. Les connecteurs et la fonction en logique n'œuvrent plus sur des individualités homogènes, mais sur des éléments différents et les opérations ne sont plus des additions ou des multiplications mais seulement des descriptions d'états de coprésence. Même si sont encore présents - dans le calcul propositionnel, dans celui des prédicats, des classes ou des relations - des symboles dont on peut traiter en faisant abstraction du signifié, tout connecteur exprime une position entre deux éléments (propositions, termes, actions) parmi les seize possibles (dans la logique bivalente que nous savons être à la base du calcul combinatoire). En reprenant la présentation de BochenskiMenne, nous pouvons dire :

«Signification du contenu des foncteurs dyadiques des valeurs de vérité. »

\begin{tabular}{|c|c|c|}
\hline Matrix & Nom & Contenu de signification \\
\hline 1111 & Tautologie & Tout (vaut dans tous les cas) \\
\hline 1110 & Disjunktion & Au moins un (non aucun) \\
\hline 1101 & Replikation & L'autre non sans l'un \\
\hline 1100 & Präpendenz & $\begin{array}{l}\text { En chaque cas l'un } \\
\text { (indédifférement aussi l'autre) }\end{array}$ \\
\hline 1011 & Implikation & L'un non sans l'autre \\
\hline 1010 & Postpendenz & $\begin{array}{l}\text { En chaque cas l'autre } \\
\text { (indifféremment aussi l'un) }\end{array}$ \\
\hline 1001 & Äquivalenz & $\begin{array}{l}\text { Non un tout seul } \\
\text { (les deux ou aucun) }\end{array}$ \\
\hline 1000 & Konjunktion & Les deux \\
\hline 0111 & Exklusion & Un au plus (pas les deux) \\
\hline 0110 & Kontravalenz & $\begin{array}{l}\text { Uniquement un des deux } \\
\text { (soit l'un, soit l'autre) }\end{array}$ \\
\hline 0101 & Postnonpendenz & $\begin{array}{l}\text { En aucun cas l'autre } \\
\text { (indifféremment l'un) }\end{array}$ \\
\hline
\end{tabular}

${ }^{11}$ [Boole 1976, 45]. 


\begin{tabular}{lll} 
Matrix & Nom & Contenu de signification \\
\hline 0100 & Postsektion & $\begin{array}{l}\text { L'un sans l'autre } \\
\text { En aucun cas l'un } \\
\text { (indifféremment l'autre) }\end{array}$ \\
0011 & Pränonpendenz & L'autre sans l'un \\
0010 & Präsektion & Aucun (non les deux) \\
0001 & Rejektion & Rien (ne vaut en aucun cas) \\
0000 & Antilogie & \\
\hline
\end{tabular}

De fait, autant le calcul propositionnel que celui des prédicats, des classes et des propositions ou des termes ou des classes se taisent au sujet du contenu conceptuel, alors que la logique aristotélicienne-thomiste entendait évaluer des termes et des attributions relatives (on se souvient par exemple de l'attribution par soi ou par accident du prédicat au sujet). Mais les calculs des propositions, des prédicats, des relations et des classes, en logique symbolique affrontent le problème des modes d'association entre deux éléments au moins, et c'est là qu'ils saisissent l'essence du rapport présenté par tout connecteur et le définissent. Nous pouvons alors dire que la logique symbolique moderne avec les seize connecteurs (dans le cas de la logique bivalente) approfondit un type de rapport : conjonction, alternative, équivalence, implication matérielle ... ainsi le même terme « connecteur » désigne opportunément un rapport dans lequel deux éléments sont précisément rapprochés. La logique aristotélico-thomiste examine d'autres aspects (attribution nécessaire ou contingente, analogie ou univocité ou équivocité des termes ... ). Prenons un exemple commode pour les deux logiques :

«cet homme est ensemble un lion et un taureau»

«cet autre est parfois mouton ou ours ».

Taureau, lion, mouton ou ours représentent des attitudes (respectivement la force, le courage, la faiblesse ou l'extrême réserve), ce qui suppose la doctrine de l'analogie de la logique aristotélico-thomiste. En revanche, on a besoin des connecteurs que nous fournit la logique symbolique pour réfléchir sur la signification du fait d'être taureau et lion ensemble, mouton ou ours. En d'autres termes, la logique aristotélicienne est une logique «nucléaire » : compréhension du terme, du concept, recherche sur l'attribution du prédicat au sujet ... La logique symbolique moderne, en revanche, à partir de deux éléments dans le calcul bivalent (ou de plus dans le calcul trivalent ...) tend à connaître les possibles états de

\footnotetext{
${ }^{12}$ [Bochenski 1983, 35].
} 
présence dans l'être et dans l'action : c'est ici un problème de coordination ou de coprésence de caractérisations ou de remarques. Et justement parce qu'elle exprime la simple coordination, le fait de la coprésence, elle n'exprime pas pourquoi intervient l'accord, ni le quand, ni le comment ... et n'éclaire pas non plus sur le fait de savoir si la remarque ou la caractérisation en question est provisoire, accidentelle ou permanente ou essentielle. Il est donc seulement question de coprésence factuelle.

Pour une confirmation ultérieure il est utile de rappeler que certaines opérations valides en logique symbolique sont erronées en arithmétique; on pense à la dualité, à l'équivalence ou à la propriété distributive, qui sont bien différentes dans les deux sciences. Et en outre alors qu'en mathématique :

$$
\begin{gathered}
1+1=2 \\
1 \times 1=1 \\
1 \times 2=2 \\
1 \neq 0
\end{gathered}
$$

Boole comprend qu'en logique :

$$
\begin{array}{cl} 
& 1+1=1 \\
1 \times 1=1 \\
1 \times 2 \quad & \text { n'a pas de sens } \\
1=-0 \\
0=-1
\end{array}
$$

pourvu qu'on donne à un la valeur de classe universelle et à 0 la valeur de classe nulle. Il est évident que l'universalité + l'universalité = l'universalité, que le deux n'a pas de sens, et qu' 1 et 0 se contredisent si bien que $1=-0$ et $0=-1$. Ainsi il est opportun de changer de symboles : $\wedge$ et $\vee$, par exemple, où $\wedge$ indique proprement la communauté et $\vee$ indique l'appartenance à un des groupes en question ou aux deux. Il y a des hommes qui sont commerçants et des hommes qui sont grands : les commerçants qui sont grands sont exprimés par $\wedge$. Le signe $\vee$ exprime en revanche les hommes qui sont ou commerçants, ou grands, ou les deux.

Mais $\wedge$ et $\vee$ ne sont pas équivalents ou convertibles avec $\times$ ou + . Les opérations rationnelles sur les nombres ont comme présupposé fondamental que les données des opérations sont homogènes : il n'est pas possible d'additionner ou de soustraire des objets différents. Un homme 
ne peut s'additionner avec une chaise, une ville ne peut se diviser par un cadre. En outre le résultat égalera parfaitement les facteurs en jeu : $2+3=5$ ou $2 \times 3=6$, sans qu'il reste rien. Il en va ainsi aussi dans la soustraction : $6-3=3$, ou dans la division : $10: 2=5$ ou encore $11: 2=5$, avec le reste de 1 .

Ceci ne se produit pas avec les connecteurs logiques. La conjonction admet la coprésence d'éléments même différents : par exemple commerçants et grands. Le résultat n'égale pas non plus parfaitement les éléments de départ. Les commerçants et les grands hommes continuent à exister dans leur autonomie, ils ne se dissolvent pas dans le 6 comme le 3 et le 2 multipliés, mais ils s'unissent seulement dans le sens où ils sont ensemble dans une situation déterminée.

Nous pouvons encore préciser : le connecteur logique $\vee$ (vel latin, l'alternative) indique que la fausseté subsiste dans l'hypothèse où les deux éléments de départ ( $p$ et $q$ ) sont l'un et l'autre faux et que la conclusion est vraie si l'un des deux éléments ou les deux sont vrais. Mais ceci n'est pas l'addition. « Pour participer au concours une licence ou une expérience professionnelle est nécessaire », dans cette affirmation il est clair qu'il y a trois possibilités, car la participation au concours est conditionnée par la possession ou d'une licence ou d'une expérience professionnelle ou des deux . L'addition est une chose bien différente : la somme résulte de l'unification des deux éléments de départ (le 5 unifie le 3 et le 2 ), alors que la licence ou l'expérience professionnelle ou les deux ne fusionnent nullement, mais indiquent seulement qu'un élément est suffisant afin de se présenter au concours ou la possibilité des deux éléments.

Nous pouvons résumer :

- en arithmétique $(2+5=7) \times(1+3=4)=28$ en logique symbolique $(2+5=7) \wedge(1+3=4)=1$

- en arithmétique $(2+5=7)+(1+3=4)=11$ en logique symbolique $(2+5=7) \vee(1+3=4)=1$

- en arithmétique $(2+5=7)+(1+3=1)=8$ : le résultat est erroné parce que l'opération dans la seconde parenthèse est erronée ; en logique symbolique $(2+5=7) \vee(1+3=1)=1$ : le résultat est exact bien que l'opération dans la seconde parenthèse soit erronée.

La raison profonde de tout le raisonnement tient précisément dans le fait que la fonction de ces opérations logiques n'est pas additionnelle ou non, cumulative ou non, mais seulement de coprésence et de connexion des caractérisations (ou non). Le discours s'élargit quand 1 et 0 n'indiquent plus la classe universelle ou la classe nulle, mais positif ou négatif, vrai et 
non-vrai, bon ou non-bon ... et si l'on admet la possibilité d'une graduation : $1,1 / 2,0 ; 1,4 / 5,3 / 5,2 / 5,1 / 5,0 ; \ldots$ Mais une logique polyvalente ou seulement trivalente peut recevoir des aspects variés, sur la base du sens à attribuer aux possibilités intermédiaires :

a) gradualité des valeurs : ex. gradualité de l'intelligence, de beauté

b) probabilité : prévision mathématique/prévision floue

c) propriétés matérielles; poids, hauteur ...

De cette manière, les valeurs $4 / 5,3 / 5 \ldots$ peuvent signifier et ont été interprétées de fait comme possibilités moyennes, futur contingent, paradoxal, inconnu, peu sûr, probable $\ldots{ }^{13}$ Mais s'il est question de valeurs en un sens étroit (comme dans le cas de la gradualité de l'intelligence, de la beauté, comme aussi quand il est question de dignité, de découvertes, d'inventions ...), le discours se présente de manière complètement différente, puisqu'on entre alors dans une logique de la subsomption : deux chevaux, dont chacun par exemple peut tirer 20 quintaux, en tireront ensemble 40 : leurs forces s'additionnent. Deux inventions de même type, dont la première possède une validité de degré 10 et la seconde de degré 5 ne s'additionnent pas : en ce cas $10+5=10$. Emerge ainsi une logique de l'analogie bien différente de la mathématique et de la logique de Boole-Frege. Une autre considération fondamentale est donnée par le fait que le calcul trivalent peut assumer tout calcul polyvalent : en fait au lieu de $1,4 / 5,3 / 5,2 / 5,1 / 5$, on pourrait utiliser seulement $1,1 / 2,0$ pour que cette dernière expression devienne « Supérieur, Moyen, Moindre (qui peut s'adapter, par exemple, à 4/5, 3/5, 2/5, ou bien encore à $3 / 5,2 / 5,1 / 5)$. Au même moment on soulignera le fait que 1 ( ou $V$ ) indique la plénitude de la vérité, de la validité ... ce qui n'est pas identifiable avec la « généralité » dont la science fait un large usage, généralité avec laquelle il est impossible de former le carré logique parce que manquent les valeurs universelles affirmatives et négatives. La catégorie de généralité sera plutôt réduite à la catégorie de «Maximum, Moyen, Minimum », mais dans ce cas on aurait :

Maximum $=$ probabilité maximale, extension maximale, non dans l'absolu mais relativement à un domaine donné : par exemple, $90 \%$. Moyen $=$ probabilité moyenne, toujours relativement ..., par ex. $50 \%$.

Minimum $=$ probabilité minime, extension minime, toujours relativement ..., par ex. $10 \%$.

Ainsi Blanché note-t-il exactement la différence entre :

${ }^{13}$ Voir ici [Rescher 1969, 23-41]. 
a) certitude et incertitude $=$ probabilité de la loi (degré de confirmation d'une hypothèse, de Carnap) et

b) contenu probable, contenu mesuré (fréquence relative d'une grandeur, chez Carnap).

Dans le premier cas, on a une triade exhaustive : nécessaire-possiblecontingent-impossible; dans le second cas, on a un éventail de situations : nécessaire, $-4 / 5$ de probabilité, $-3 / 5,-2 / 5,-1 / 5$ de probabilité impossible ${ }^{14}$.

B) Voici encore quelques réflexions sur la fonction. Dans son commentaire sur le célèbre passage de Frege sur le concept de fonction Bochenski souligne : «il introduit, précisément, trois concepts fondamentaux : 1) celui d'argument, et de lieu d'argument; 2 ) celui de valeur (d'une fonction ) ; 3) celui de fonction «non saturée », c'est-à-dire contenant une variable $^{15}$. D'où la fonction propositionnelle de Russell ${ }^{16}$.

Quels peuvent bien donc être les avantages et les limites de cette imposition? L'avantage de la fonction, à l'exemple des autres correspondances, réside dans l'unicité de l'image du domaine commun : un - un, plusieurs - un. Au contraire dans les autres correspondances (plusieurs plusieurs ; un - plusieurs) il y a une pluralité d'éléments dans le domaine commun, c'est-à-dire une dispersion des indications.

Mais le présupposé est qu'à l'x se substituent des nombres (Frege dit clairement que nous sommes dans le règne de la somme, du produit, de la puissance ...). Il est possible de substituer à $\left(2+3 \cdot x^{2}\right) \cdot x$, dans l'exemple de Frege, 0,1, 2,3 pour obtenir respectivement 0, 5, 28, 87, mais il n'est pas possible de substituer à $x$, toujours dans $\left(2+3 \cdot x^{2}\right) \cdot x$, un nom de personne ou de ville. Mais la fonction n'est pas seulement celle de la mathématique. La correspondance automobile - numéro de plaque (minéralogique), comme la correspondance personne - quotient d'intelligence sont des fonctions. Il y a un rapport un-un dans le premier cas, plusieurs-un dans le second, mais de toute façon le rapport n'est pas entre des nombres, il est entre des réalités déterminées sur la base d'un critère, de reconnaissance officielle d'appartenance dans le premier cas, de degré de capacité intellectuelle dans le second. Ce critère pourra être très variable : filiation-paternité, propriété des biens, causalité efficiente ... En tous ces cas il y a non pas homogénéité (par définition) du nombre, comme dans l'exemple donné par Frege, mais un rapport déterminé de causalité ou d'exemplarité (modèle) ou de tendance ..., un rapport qui

\footnotetext{
14 [Blanché 1975, 133-138].

15 [Bochenski 1972, 418].

${ }^{16}$ [Bochenski 1972, 418-419].
} 
explique précisément la constitution de la fonction, et que nous pourrons bien appeler d'homogénéité au sens large ou d'affinité (d'analogie? Mais de cela il sera question peu après).

Dans le même moment se trouve souligné le fait que la fonction n'explicite pas le motif de cette homogénéité au sens propre ou impropre (affinité) : elle affirme ce qui est. Dans le cas du rapport automobilenuméro de plaque (minéralogique), il est suffisant d'en appeler, comme preuve, à la reconnaissance officielle; dans le cas du quotient intellectuel il suffit d'en appeler à la mesure expérimentale : la fonction ne va pas audelà, pour tenter de rechercher les causes, les modalités, les conditions, les nécessités ou non, de l'attribution. Et cela parce qu'une telle exigence d'enrichissement conceptuel peut être satisfaite non pas sur la base de la connaissance de l'existence présentée par la fonction, mais sur la base de la compréhension de ce qui est essentiel et constitutif du fait; mais c'est précisément cela qui ouvrirait la fonction à de nouvelles perspectives.

En résumant, donc, la fonction prend la forme d'une relation ad unum, homogène, et de fait.

C) Ici il est opportun de consacrer un bref développement au problème de l'analogie

- Nous pouvons partir de quelques lois logiques. Que l'on pense, par exemple, au principe d'identité qui s'exprime dans le calcul propositionnel par $p \equiv p$ ou bien Epp et dans le calcul des classes $a=a$ (le principe de non contradiction et le principe du tiers exclu s'expriment de manière similaire, avec les différenciations nécessaires). A présent il est facile de constater qu'un principe d'identité, formulé comme ci-dessus, devient tautologique et superflu, tout à fait infécond. Dire $p \equiv p$ ou $a=a$ signifie dire eau=eau, pierre=pierre. Est-ce que cela est utile à la science? Le principe d'identité exprime précisément l'identité entre un contenu et ses constituants de telle manière que soit fondée l'affirmation $4=2+2$ ou "eau $=$ $\mathrm{H}_{2} \mathrm{O}$ " ou encore "homme = animal rationnel" (au moment où on découvre la réalité du second terme). Pour un tel cas, il y aurait besoin d'inventer une symbolique adéquate pour le second terme, qui explicite précisément les constituants du premier terme et ne le répète pas seulement. Et cela vaut évidemment pour toute expression de science expérimentale, de mathématique, du langage ordinaire ...

- Que l'on pense encore au terme "cause". Il y a toute sorte de types de causes : cause efficiente (le menuisier qui construit un meuble), finale (mes intentions), exemplaire (le modèle), cause principale (le peintre qui peint) ou instrumentale (le pinceau dans la main du 
peintre), cause du commencement ou cause de la conservation de quelque chose, cause génératrice ou au contraire productrice ... Et, précisément pour satisfaire les exigences universellement réclamées de précision, comment ne pas invoquer un symbolisme différencié?

- Il est inutile de souligner que le terme « liberté » est également analogue (liberté naturelle ou libre arbitre, liberté morale, politique, économique, syndicale, de conscience ... ), de même que les termes "bien", "morale", "unité", "justice", "histoire" ...Que l'on pense à puissance passive, active, objective; à la loi naturelle, scientifique, juridique; au droit subjectif, objectif, naturel, positif; à l'infini, potentiel et actuel. Blanché écrit : «L'usage de l'article « déterminé » est aussi équivoque que celui de l'article « indéterminé », puisqu'il annonce tantôt le genre ( «le cheval est la plus belle conquête de l'homme») tantôt l'individu («le cheval a fait un écart en arrière »). La copule « est »a, pour sa part, des sens multiples; inhérence d'un attribut à un sujet, ( « Pierre est homme»), appartenance d'un individu à une classe ( « Pierre est un homme»), implication d'un attribut par un attribut ( «les artistes sont susceptibles »), inclusion d'une classe dans une classe ( « les mammifères sont des vertébrés»), équivalence soit affirmée ( « Paris est la capitale de la France ») soit simplement posée à titre de définition ( « le cercle est le lieu des points ...»); sans parler des usages noncopulatifs de ce même terme : auxiliaire des temps (《il est parti ») ou des formes du verbe (《il est aimé »), verbe qui indique l'existence (parfois difficilement distinct d'une copule : « homo mendax est » peut signifier que «l'homme est menteur » ou qu' «il existe un homme menteur »), etc. La syntaxe est trompeuse comme le vocabulaire. Considérons par exemple le cas de l'adjectif épithète. Celui que nous appelons «un peintre italien » unit les deux attributs et est en même temps peintre et italien. Mais « un peintre accompli » n'est pas accompli absolument, il l'est seulement en tant que peintre. Un peintre «abstrait »n'est abstrait d'aucune manière, c'est seulement sa peinture qui l'est (cf. un blessé grave, une paralysie générale, etc.). Enfin « un peintre raté »n'est plus peintre du tout (cf. un futur ministre, un candidat éventuel, un présumé coupable ...). On peut multiplier à l'infini les exemples de semblables fluctuations dans les langues naturelles $»^{17}$.

- Que l'on pense aux divers sens du « est» (nous relevons un texte de Bochenski-Menne) : «Le mot allemand « ist » (et les mots correspondants dans les autres langues européennes) comprend deux

${ }^{17}[$ Blanché 1968, 10]. 
groupes de significations très différentes : des significations existentielles et des significations copulatives :

« Le mot « ist » a, entre autres, deux significations existentielles :

« Existence d'un objet bien caractérisé.

« Non viduité d'une classe.

Les deux sont définies à l'aide de l'opérateur existentiel.

« Le mot « ist »a, entre autres, quatre significations copulatives :

« L'ordination d'un prédicat à un individu.

« L'appartenance d'un élément à une classe.

« L'inclusion d'une classe dans une autre.

« L'identité ${ }^{18}$.

- Que l'on pense ainsi à la «supposition »des termes ${ }^{19}$ :

homme est un mot de cinq lettres (supp. matérielle)

homme est un concept universel (supp. formelle-logique)

l'homme est un animal social (supp. formelle-réelle)

l'homme a une âme spirituelle (supp. réelle-essentielle)

l'homme se rend au travail (supp. réelle-personnelle)

l'homme est peintre, musicien (supp. discrète-personnelle)

l'homme vit à Paris (supp. discrète-déterminée)

l'homme désire vivre (supp. discrète-confuse).

- Que l'on pense encore au champ très ample de l'analogie et de l'équivocité dans le langage ordinaire ${ }^{20}$. " $x$ est un chien " peut signifier :

"Fido est un chien"

"le Cervin est un chien"

"Le professeur N. N. est un chien".

Mais on comprend facilement que le premier sens est Vrai au sens propre, le second est Faux, et le troisième peut être vrai en un sens analogue. "x est capitale" peut signifier :

"Rome est capitale (d'Italie)"

"un milliard est capital (de la société)"

"l'entreprise des Mille est capitale (pour le Risorgimento italien). Mais il est clair que le terme "capital", dans les exemples adoptés, est un terme analogue.

Il en va de même dans le syllogisme, par exemple :

\footnotetext{
${ }^{18}$ [Bochenski 1972, 84-85].

${ }^{19}$ Notée en substance par S. Vanni Rovighi [Rovighi 1962, 64-65].

${ }^{20} \mathrm{Il}$ est utile de se souvenir que nous entendons par terme ou concept «analogue » un terme (ou concept) susceptible d'être prédiqué d'objets variés de manière non parfaitement identique (par exemple, «mouton» dit de l'animal ou d'un homme timide), et par « équivoque » le terme (jamais le concept) prédiqué de manière complètement différente (par exemple la « flèche » projectile et constellation, cf. ci-dessous).
} 
« La flèche est la constellation située au nord d'Altaïr

La flèche est un projectile qui blesse

Donc la constellation située au nord d'Altaïr est un projectile qui blesse »

où il est évident que le terme «flèche » a un double sens, complètement différent (c'est un terme équivoque) et ici le syllogisme en vient à avoir quatre termes et non trois.

- On se permettra une vaste exemplification dans le seul but de montrer l'ampleur de l'analogie qui dans le langage humain et dans la pensée n'a pas en fait une présence marginale ou exceptionnelle. On ne peut non plus accuser ce langage d'ambiguïté en un sens péjoratif (c'est-à-dire erroné ou dangereux) : ambiguïté signifie seulement en ces cas-là duplicité de signification sur la base précisément de l'analogie ou de l'équivocité : lion, agneau, taureau, serpent, colombe, ours, limace, tortue, fourmi, aigle, renard, faucon, chien de mer, baleine, chien, chouette, cigogne, félin, ..., sapin, courge, navet, épinards, chrysanthème, ... roche, tremblement de terre, foudre, tempête. Ainsi encore pour les corps célestes : bélier, scorpion, gémeaux, soleil ...; pour les nombres dans la Bible (soixante dix fois sept fois; les âges des patriarches, le 7 , le 12, le $40 \ldots$...), dans les cartes de jeu ou les sociétés secrètes.

- Blanché écrit «C'est seulement quand on sera parvenu à une sécurité absolue de la correction logique des formes grammaticales en usage qu'on pourra les prendre comme substituts des formes logiques elles-mêmes. Alors il sera permis d'oublier totalement le contenu des énoncés et ce non seulement le sens intuitif des termes qui peuvent occuper la place des variables, comme le faisait la logique classique, mais également le sens des locutions proprement logiques, comme «tout», «si... alors », etc. En effet, si les symboles qui les représentent ont eu leur grammaire rigoureusement et explicitement formulée, il suffira, pour utiliser correctement ces symboles, de se conformer aux règles $\gg^{21}$.

Mais, d'autre part, le calcul ne se laisse pas comprendre comme une mécanique, puisqu'il n'en est pas une. C'est une œuvre de cette intuition dénigrée ou intellection immédiate. L'exemplification opérée par le symbolisme et le calcul apporte sans conteste une clarté expressive (encore que restreinte et particulière comme on l'a vu) mais cette clarté doit être comprise accompagnée d'une intuition dont la limpidité et la sûreté viendront sans aucun doute précisément de cette clarté expressive. Mais le symbolisme et le cal-

${ }^{21}$ [Blanché 1968, 10]. 
cul restent de purs instruments de l'intuition, et ne la remplacent pas : ce n'est pas pour rien que l'enregistreur répète et relie sans l'intelligence de la répétition. L'intuition soutient tout acte humain intelligent.

L'amplitude donnée au problème de l'analogie et de l'équivocité dépend de son ampleur effective dans la réalité du langage et de la pensée humaine. Et, si nous sortons du point de vue logique, il semble qu'une telle ampleur soit due à la l'étendue des rapports de confiance qui semblent beaucoup être plus larges que les conquêtes scientifiques. Que l'on pense aux rapports entre père et fils, mari et femme, entre amis, entre éducateurs et disciples, entre médecins et patients ... : la confiance est présente également là où il y a des preuves scientifiques, puisque celles-ci en général sont seulement hautement probables (la science expérimentale elle-même ne se proclame pas absolument certaine) et dans la majeure partie des cas ne sont pas comprises des utilisateurs (je n'ai pas la possibilité de comprendre la valeur du plan de l'édifice que j'habite ou la qualité suffisante du travail réalisé par l'entreprise constructrice, ou encore la capacité du pilote de l'avion ou du conducteur de l'autobus que j'utilise). Avec ces premiers pas de logique symbolique on peut considérer trois conséquences relevantes

1) avec une logique de la coprésence se trouve implicitement désavouée l'unicité de l'interprétation homogène du réel et la démonstration de l'insuffisance du nombre pour expliquer totalement le réel;

2) mais en même temps on peut se demander si la logique des connecteurs et de la fonction, qui pourtant entend user du symbolisme et des procédures mathématiques, est encore une logique mathématique, à partir du moment où elle est une logique de la coprésence;

3) et enfin si la logique symbolique ainsi conçue ne nous montre pas clairement ses propres limites en ignorant l'analogie et l'équivocité.

III. Avec l'informatique on dépasse l'ambiguïté (ou mieux la variété et la richesse) de la langue d'une part et l'exactitude formelle du nombre de l'autre ${ }^{22}$, mais on a un langage binaire qui traduit, par le moyen de codes,

\footnotetext{
${ }^{22}$ Sur ce point, de manière plus détaillée, voir [Bozzi 1996, 87-93]. Les langues sont toutes plus ou moins complexes pour des motifs variés : poids de l'émotivité, analogies et équivocités, traditions, influences d'autres langues .... ceci jusqu'aux divers tons de prononciation de la syllabe chinoise (quatre tons en mandarin, par exemple, et neuf en cantonnais). En ce qui concerne la mathématique à l'inverse, on rencontre
} 
les diverses langues avec leurs contenus singuliers, en évitant ambiguïté et la pure formalité, en une correspondance biunivoque conventionnelle entre signe et signifié ${ }^{23}$. Sans cette correspondance biunivoque conventionnelle, il y aurait confusion de signifié : le caractère conventionnel permet d'élargir à tout domaine et à toutes les possibilités la correspondance biunivoque qui de par sa nature ne réussirait pas à conjuguer, avec complétude, la richesse de la langue et l'exactitude du signe utilisé. Et dans le même temps avec le même système binaire on a une correspondance avec les circuits électriques, et en conséquence le dépassement, au moyen de la vitesse, de la longueur des expressions.

Sans ce caractère conventionnel les connecteurs, négations, quantifications, fonctions, ne seraient que des moments d'un discours : pour dépasser le caractère fragmentaire de ces éléments entre en jeu le « code » de traduction à partir d'un langage ordinaire. Sans ce code, on ne pourrait pas dépasser le caractère fragmentaire des éléments et s'approcher de la réalisation d'un discours unitaire. On se trouverait dans la situation d'un enfant qui ne dirait que des mots isolés ou de quelqu'un qui débute tout juste l'étude d'une langue étrangère. Mais la traduction objective opérée par le code est vivifiée, complément absolument nécessaire, par le caractère conventionnel de la correspondance qui légitime, dans le contexte, le sens voulu.

En résumant : sur le présupposé indispensable des fondements de la logique de Boole et Frege l'informatique se constitue grâce à l'action du code, de la correspondance biunivoque conventionnelle et du circuit : le code permet le passage des instruments élaborés par Boole et Frege à un langage aussi vaste que l'on veut; la correspondance biunivoque entre signe et signifié, bien que conventionnelle, permet la permanence du signifié conceptuel et expressif même dans le cas d'ambiguïté du terme; le circuit annule la difficulté de l'énorme masse de bits que l'on produit avec la traduction de tout discours en langage binaire ${ }^{24}$. Sur ces bases se sont constituées les structures porteuses de la mentalité informatique, qui probablement s'explicite par :

a) le temps réel, qui dépasse les distances spatio-temporelles : d'où la relative uniformité du langage et la relative globalisation de base. Negroponte écrit : « La dite autoroute de l'information vise à transporter

les problèmes posés par l'infinité en puissance. Dans la réalité existent des choses uniques, ou deux ou trois, comme aussi la moitié, le quart ..., mais de toute façon il y a la possibilité de construire la série d'une multiplicité indéfinie de nombres, et l'indéfini multiplie les difficultés ...

${ }^{23}$ Voir [Busa 1987, 44].

${ }^{24}$ Il est évident que d'autres instruments encore (fibres optiques, satellites), que nous ne mentionnons pas ici, sont nécessaires pour la transmission des données. 
dans toute la planète du bit sans poids à la vitesse de la lumière. C'est une période pendant laquelle toute entreprise se regarde dans le miroir en s'interrogeant sur son futur dans un monde digital : eh bien, ce futur dépend presque à cent pour cent de la capacité de l'entreprise à traduire sous forme digitale ses produits et ses services $\gg^{25}$.

b) l'internet (bibliothèque électronique, encyclopédie électronique....) avec un relatif élargissement de l'information, abondance effrayante des moyens mis à disposition : d'où la possibilité d'un ample esprit critique ${ }^{26}$.

c) la multimédiatisation; «Les produits multimédiatiques incluent aussi bien la télévision que les ordinateurs avec possibilité de vidéo. Comme il a déjà été dit, la différence entre eux est subtile et le deviendra de plus en plus, peut-être jusqu'à disparaître ${ }^{27}$. «Le concept de multimédia inclut nécessairement des idées comme le fait de passer facilement d'un moyen à un autre, de dire la même chose selon des modes divers, d'avoir recours, selon les cas, à l'un ou à l'autre de nos sens : si vous n'avez pas compris ce que je vous ai dit la première fois, permettez que moi (la machine) je vous l'illustre par un dessin animé ou un diagramme tridimensionnel ... $»^{28}$.

d) la simulation, par laquelle l'homme peut essayer de nouvelles voies en en examinant les conséquences. Plutôt qu'essayer par réussites et échecs dans l'expérience on construit, on corrige et vérifie un modèle hypothétique, mais concret. Les avantages sont évidents lorsqu'on pense aux limites imposées par la réalité objective et au contraire à la liberté de l'imaginaire, même si existe pourtant le danger, à éviter, d'envolées excessives $^{29}$.

Pour tous ces motifs l'informatique n'est pas la continuation ou l'intensification de la première et de la seconde technique comme aussi celle de l'époque moderne, mais innovation et saut. Il n'est pas inutile de souligner que le caractère conventionnel de la correspondance ouvre la rigueur et la précision de la logique symbolique au vaste océan de l'être. L'homme informatique est plus voisin des valeurs qu'on ne le suppose : la technologie informatique n'est pas seulement ordre et rationalisation des processus, comme la première et la seconde technique, mais aussi

\footnotetext{
${ }^{25}$ [Negroponte 1999, 2].

${ }^{26}$ [Lewis 2002, 137-140, 187 ss.].

27 [Negroponte 1999, 68].

28 (voir [Negroponte 1999, 70]).
}

29"Un modèle numérique n'est pas lu ou interprété comme un texte classique, il est le plus souvent exploré de manière interactive. Contrairement à la plupart des descriptions fonctionnelles sur papier ou aux modèles réduits analogiques, le modèle informatique est essentiellement plastique, dynamique, doué d'une certaine autonomie d'action et de réaction" [Lévy 1990, 137]. 
tendance à atteindre consciemment le tout.

\section{Références}

Abbagnano, Nicola

1964 Dizionario di filosofia, Torino, UTET, voce Matematica, 543546, 1964.

Arnauld, A. \& Nicole, P.

1969 Logica o arte di pensare, in Grammatica e logica di Port-Royal, Roma : Ubaldini, 104, 1969.

BELL, D.

1976 The coming of Post-industrial Society. A venture in Social Forecasting, London : Harper Collins, 9, 1976.

BlAnChÉ, R.

1975 L'induction scientifique et les lois naturelles, Paris : PUF, 133138, 1975.

1968 Logica e assiomatica, Firenze : La Nuova Italia, 10, 1968.

Bochenski, J. M.

1972 La logica formale. La logica matematica, Torino : Einaudi, 1972.

Bochenski, J. M. (erweitet von A. Menne),

1983 Grundriß der formalen Logik, 5 ed., Paderborn : Ferdinand Schöningh, 35, 1983.

Boole, G.

1976 Indagine sulle leggi del pensiero, Torino : Einaudi, 1976.

Bozzi, R.

1996 Introduzione al futuro. Ipotesi sulle radici della scienza dell'informazione, 2 ed., Bari : Adriatica editrice, 87-93, 1996.

Busa, R.

1987 Fondamenti di informatica linguistica, Milano : Vita e pensiero, 44, 1987.

Gerhardt, Carl Immanuel (éd.)

1849-1855 Leibnizens mathematische Schriften, III, Berlin : Asher, 53, 1849-1855.

Kalinowski, Georges

1974 Raison, entendement et philosophie, Dialogue, 13, Montréal : 123-138, 1974. 
LENoble, R.

1969 Le origini del pensiero scientifico moderno, in Vari, Storia della scienza (a cura di M. Daumas), I, Bari : Laterza, 381-382, 1969.

LEWIS, M.

2002 Next. Il futuro è già avvenuto, Roma : Editori Riuniti, 137-140 et 187, 2002.

LÉvY, P.

1990 Les technologies de l'intelligence. L'avenir de la pensée à l'ère informatique, Paris : La Découverte, 137, 1990.

LOCKE, J.

1951 Saggio sull'intelligenza umana, I, Bari : Laterza, 123-125, 1951. Negroponte, N.

1999 Essere digitali, 2 ed., Milano : Sperling \& Kupfer ed., 2, 1999. Odifreddi, Piergiorgio

2000 La matematica del Novecento. Dagli insiemi alla complessità., Torino : Einaudi, 26, 2000.

RESCHER, N.

1969 Many-valued logic, New York : McGraw-Hill Inc., 23-41, 1969. VAnni Rovighi, S.

1962 Elementi di filosofia, I, Introduzione. Logica. Teoria della conoscenza, Brescia : La Scuola, 64-65, 1962. 